\title{
INVARIANT SUBSPACES FOR CLOSED $*$-REPRESENTATIONS OF *-ALGEBRAS
}

\author{
ITSUKO IKEDA AND ATSUSHI INOUE
}

(Communicated by Palle E. T. Jorgensen)

\begin{abstract}
The first purpose of this paper is to investigate the selfadjointness of $*$-subrepresentations of closed $*$-representations. The second purpose is to define the notion of selfadjoint vectors for any closed $*$-representation $\pi$ of a *-algebra $\mathscr{A}$ and to show that $\pi$ is decomposed into $\pi=\pi_{1}^{s} \oplus \pi_{2}^{s}$, where $\pi_{1}^{s}$ is a direct sum of cyclic selfadjoint representations of $\mathscr{A}$ and $\pi_{2}^{s}$ is a closed *-representation of $\mathscr{A}$ that does not have any nonzero selfadjoint vector.
\end{abstract}

\section{INTRODUCTION}

Unbounded *-representations of *-algebras have been studied by Gudder, Jorgensen, Powers, Schmüdgen, etc. from the mathematical interest and from situations of the physical applications. As Powers pointed out in [6], there are some pathologies for $*$-subrepresentations of unbounded $*$-representations. Let $\pi$ be a closed $*$-representation of a $*$-algebra $\mathscr{A}$ on a Hilbert space $\mathscr{H}(\pi)$. For each $\pi$-invariant subspaces $\mathscr{E}$ of the domain $\mathscr{D}(\pi)$, the closure $\pi_{\mathscr{E}}$ of the restriction of $\pi$ to $\mathscr{E}$ is a closed *-representation of $\mathscr{A}$ on the Hilbert space $\overline{\mathscr{E}}$. But the projection $P_{\overline{\mathscr{E}}}$ of $\mathscr{H}(\pi)$ onto $\overline{\mathscr{E}}$ does not necessarily belong to the weak commutant $\pi(\mathscr{A})_{\mathrm{w}}^{\prime}$ of the $O^{*}$-algebra $\pi(\mathscr{A})$. When $P_{\overline{\mathscr{E}}} \in \pi(\mathscr{A})_{\mathrm{w}}^{\prime}$, we have another *-representation $\pi_{P_{\overline{\mathscr{E}}}}$ of $\mathscr{A}$, and $\pi_{\mathscr{E}} \subset \pi_{P_{\overline{\mathscr{E}}}}$, but $\pi_{\mathscr{E}} \neq \pi_{P_{\bar{\varepsilon}}}$, in general. Then we study the relation between $\pi_{\mathscr{E}}$ and $\pi_{P_{\bar{\varepsilon}}}$ in detail, and the selfadjointness of these two *-representations. In case $\pi$ is selfadjoint, Powers has obtained a necessary and sufficient condition for the selfadjointness of $\pi_{\mathscr{E}}$. In $\S 3$ we obtain the result that $\pi_{\mathscr{E}}$ is selfadjoint iff $P_{\overline{\mathscr{C}}} \mathscr{D}\left(\pi^{*}\right)=\mathscr{D}\left(\pi_{\mathscr{E}}\right)$ and that $P_{\overline{\mathscr{C}}} \mathscr{D}(\pi) \subset \mathscr{D}(\pi)$ iff $P_{\overline{\mathscr{E}}} \in \pi(\mathscr{A})_{\mathrm{w}}^{\prime}$ and $\pi=\pi_{P_{\overline{\mathscr{E}}}} \oplus \pi_{I-P_{\overline{\mathscr{C}}}}$.

We next investigate the conditions under which a closed *-representation decomposes into a direct sum of some cyclic representations. In this subject, Powers has obtained that each integrable representation of a commutative *algebra that is dominated by a countably generated *-subalgebra is a direct sum of cyclic integrable representations [6]. And Schmüdgen has shown that each $\mathbf{G}$-integrable representation of the universal enveloping algebra $\mathscr{E}(\mathscr{G})$ of

Received by the editors April 1, 1991.

1991 Mathematics Subject Classification. Primary 47D40, 46K40.

Key words and phrases. Closed (selfadjoint, integrable) representation selfadjoint (integrable, bounded) vector. 
the complexification of the Lie algebra $\mathscr{G}$ of a connected Lie group $\mathbf{G}$ is a direct sum of cyclic G-integrable representations. But, in case of a general unbounded representation, as seen in Example 4.4, such decomposition is not always possible. So, we consider this problem by using the results of $\S 3$ and defining the notion of selfadjoint vectors for any closed $*$-representation $\pi$. And we have the decomposition, $\pi=\pi_{1}^{s} \oplus \pi_{2}^{s}$, where $\pi_{1}^{s}$ is a direct sum of cyclic selfadjoint representations and $\pi_{2}^{s}$ is a closed *-representation that does not have any nonzero cyclic selfadjoint vector. Further, we study this problem for some concrete representations.

\section{Preliminaries}

For a dense subspace $\mathscr{D}$ of a Hilbert space $\mathscr{H}$, let $\mathscr{L}_{c}(\mathscr{D})$ denote the set of all closable linear operators $A$ in $\mathscr{H}$ with domain $\mathscr{D}$ for which $A \mathscr{D} \subset \mathscr{D}$ and $\mathscr{L}^{\dagger}(\mathscr{D})$ the *-algebra of all $A \in \mathscr{L}_{c}(\mathscr{D})$ such that $\mathscr{D} \subset \mathscr{D}\left(A^{*}\right)$ and $A^{*} \mathscr{D} \subset \mathscr{D}$, with involution, $\dagger: A \rightarrow A^{\dagger}:=\left.A^{*}\right|_{\mathscr{D}}$. Then a $*$-subalgebra of $\mathscr{L}^{\dagger}(\mathscr{D})$ is said to be an $O^{*}$-algebra. A representation $\pi$ of an algebra $\mathscr{A}$ on $\mathscr{H}(\pi):=\mathscr{H}$ with domain $\mathscr{D}(\pi):=\mathscr{D}$ is an identity-preserving homomorphism from $\mathscr{A}$ into $\mathscr{L}_{c}(\mathscr{D})$. A $*$-representation $\pi$ of a $*$-algebra $\mathscr{A}$ on $\mathscr{H}$ is a *-homomorphism from $\mathscr{A}$ into $\mathscr{L}^{\dagger}(\mathscr{D}) . \pi$ is said to be closed if $\mathscr{D}(\pi)$ is complete with the graph topology $t_{\pi}$ that is defined by the family of seminorms $\left\{\|\cdot\|_{\pi(a)}=\|\pi(a) \cdot\|: a \in \mathscr{A}\right\}$. For a representation $\pi$, the closure of $\pi$ is the smallest closed extension of $\pi$, and the adjoint $\pi^{*}$ of $\pi$ is defined as $\mathscr{D}\left(\pi^{*}\right):=\bigcap_{a \in \mathscr{A}} \mathscr{D}\left(\pi(a)^{*}\right), \pi^{*}(a):=\left.\pi\left(a^{*}\right)^{*}\right|_{\mathscr{D}\left(\pi^{*}\right)}$, for $a$ in $\mathscr{A}$. We say that $\pi$ is selfadjoint if $\pi=\pi^{*}$, and $\pi$ is integrable (or standard) if $\pi$ is closed and $\overline{\pi\left(a^{*}\right)}=\pi(a)^{*}$, for each $a$ in $\mathscr{A}$. The weak commutant $\pi(\mathscr{A})_{\mathrm{w}}^{\prime}$ of $\pi(\mathscr{A})$ is defined by $\pi(\mathscr{A})_{\mathrm{w}}^{\prime}=\left\{C \in \mathscr{B}(\mathscr{H}),(C \pi(a) \xi \mid \eta)=\left(C \xi \mid \pi\left(a^{*}\right) \eta\right)\right.$, for each $\xi, \eta \in \mathscr{D}(\pi)\}$. It is known that $\pi(\mathscr{A})_{\mathrm{w}}^{\prime}$ is a weakly closed $*$-invariant subspace of $\mathscr{B}(\mathscr{H})$, but it need not be an algebra [6]. For a representation $\pi$ of $\mathscr{A}$, a subspace $\mathscr{E}$ of $\mathscr{D}(\pi)$ is said to be $\pi$-invariant if $\pi(a) \xi \in \mathscr{E}$, for $a \in \mathscr{A}$, $\xi \in \mathscr{E}$, and a vector $\xi$ in $\mathscr{D}(\pi)$ is said to be cyclic (resp. weakly cyclic) for $\pi$ if $\pi(\mathscr{A}) \xi$ is dense in $\mathscr{D}(\pi)$ (resp. $\mathscr{H}(\pi)$ ) with $t_{\pi}$. For a family $\left\{\pi_{\lambda}: \lambda \in \Lambda\right\}$ of representations of $\mathscr{A}$, the direct sum $\pi=\sum_{\lambda \in \Lambda} \oplus \pi_{\lambda}$ is defined as

$$
\begin{gathered}
\mathscr{D}(\pi):=\left\{\xi=\left(\xi_{\lambda}\right)_{\lambda \in \Lambda} ; \quad \xi_{\lambda} \in \mathscr{D}\left(\pi_{\lambda}\right) \quad \text { for each } \lambda \in \Lambda,\right. \\
\\
\left.\sum_{\lambda \in \Lambda}\left\|\pi(a) \xi_{\lambda}\right\|^{2}<\infty \text { for each } a \in \mathscr{A}\right\}, \\
\pi(a) \xi:=\left(\pi_{\lambda}(a) \xi_{\lambda}\right)_{\lambda \in \Lambda} \quad \text { for } a \in \mathscr{A}, \xi=\left(\xi_{\lambda}\right) \in \mathscr{D}(\pi) .
\end{gathered}
$$

\section{SELFADJOINTNESS OF $*$-SUBREPRESENTATIONS OF $*$-REPRESENTATIONS}

Let $\pi$ be a closed $*$-representation of a $*$-algebra $\mathscr{A}$ on a Hilbert space $\mathscr{H}$. For a projection $P$ in $\pi(\mathscr{A})_{\mathrm{w}}^{\prime}$, we define a $*$-representation of $\mathscr{A}$ on $P \mathscr{H}$

$$
\begin{aligned}
& \mathscr{D}\left(\pi_{P}\right):=P \mathscr{D}(\pi), \\
& \pi_{P}(x) P \xi:=P \pi(x) \xi \text { for } x \in \mathscr{A}, \xi \in \mathscr{D}(\pi) .
\end{aligned}
$$

Then it follows from [6, Lemma 4.6] that

(3.1) $\mathscr{D}\left(\pi_{P}\right) \subset \mathscr{D}\left(\pi^{*}\right)$, 
(3.2) $\pi_{P}(x) P \xi=\pi^{*}(x) P \xi$, for $x \in \mathscr{A}, \xi \in \mathscr{D}(\pi)$.

Powers has obtained the result that $\pi_{P}$ is selfadjoint whenever $\pi$ is selfadjoint in [6, Theorem 4.7]. However, $\pi_{P}$ is not selfadjoint in general. It is easy to show the following result for the selfadjointness of $\pi_{P}$ when $\pi$ is not selfadjoint.

Proposition 3.1. Let $\pi$ be a closed *-representation of $\mathscr{A}$ on $\mathscr{H}$ and $P$ a projection in $\pi(\mathscr{A})_{\mathrm{w}}^{\prime}$. Then,

(i) $P \mathscr{D}(\pi)=\mathscr{D}\left(\pi_{P}\right) \subset \mathscr{D}\left(\pi_{P}^{*}\right) \subset P \mathscr{D}\left(\pi^{*}\right)$.

Suppose $P \mathscr{D}(\pi) \subset \mathscr{D}(\pi)$; then

(ii) $\pi_{P}$ is a closed *-representation;

(iii) $\mathscr{D}\left(\pi_{P}^{*}\right)=P \mathscr{D}\left(\pi^{*}\right)$;

(iv) $\pi_{P}$ is selfadjoint iff $P \mathscr{D}(\pi)=P \mathscr{D}\left(\pi^{*}\right)$.

Let $\pi$ be a closed *-representation of $\mathscr{A}$ on $\mathscr{H}$ and $\mathscr{E}$ a $\pi$-invariant subspace of $\mathscr{D}(\pi)$. We put

$$
\begin{aligned}
& \mathscr{D}\left(\left.\pi\right|_{\mathscr{E}}\right):=\mathscr{E}, \\
& \left.\pi\right|_{\mathscr{E}}(x) \xi:=\pi(x) \xi,
\end{aligned}
$$

for each $x \in \mathscr{A}$ and $\xi \in \mathscr{D}\left(\left.\pi\right|_{\mathscr{E}}\right)$. Then $\left.\pi\right|_{\mathscr{E}}$ is a $*$-representation of $\mathscr{A}$ on $\overline{\mathscr{E}}$, whose closure is denoted by $\pi_{\mathscr{E}}$. In case $\pi$ is a bounded $*$-representation, the projection $P_{\overline{\mathscr{E}}}$ of $\mathscr{H}$ onto $\overline{\mathscr{E}}$ is contained in $\pi(\mathscr{A})_{\mathrm{w}}^{\prime}\left(=\pi(\mathscr{A})^{\prime}\right)$; thus $\pi_{P_{\overline{\mathscr{E}}}}$ is well defined and equal to $\pi_{\mathscr{E}}$. But this is not true for unbounded representations $\left[6\right.$, p. 96]. Hence, we consider the relation between the $*$-representations $\pi_{P_{\overline{\mathscr{\varepsilon}}}}$ and $\pi_{\mathscr{E}}$ under the condition that $P_{\overline{\mathscr{E}}}$ is contained in $\pi(\mathscr{A})_{\mathrm{w}}^{\prime}$. First we have the following results for $\pi_{\mathscr{E}}$.

Lemma 3.2. Let $\mathscr{E}$ be a $\pi$-invariant subspace of $\mathscr{D}(\pi)$. Then

(i) $\mathscr{D}\left(\pi_{\mathscr{E}}\right) \subset P_{\overline{\mathscr{E}}} \mathscr{D}(\pi) \subset P_{\overline{\mathscr{E}}} \mathscr{D}\left(\pi^{*}\right) \subset \mathscr{D}\left(\pi_{\mathscr{E}}\right)^{*}$;

(ii) $\left(\pi_{\mathscr{E}}\right)^{*}(x) P_{\overline{\mathscr{E}}} \xi=P_{\overline{\mathscr{E}}} \pi^{*}(x) \xi$ for $x \in \mathscr{A}, \xi \in \mathscr{D}\left(\pi^{*}\right)$.

Proof. Take arbitrary $x \in \mathscr{A}$ and $\xi \in \mathscr{D}\left(\pi^{*}\right)$. For each $\eta \in \mathscr{E}$, we have

$$
\begin{aligned}
\left(\pi_{\mathscr{E}}\left(x^{*}\right) \eta \mid P_{\overline{\mathscr{E}}} \xi\right) & =\left(\pi\left(x^{*}\right) \eta \mid P_{\overline{\mathscr{E}}} \xi\right)=\left(\pi\left(x^{*}\right) \eta \mid \xi\right) \\
& =\left(\eta \mid \pi\left(x^{*}\right)^{*} \xi\right)=\left(\eta \mid P_{\overline{\mathscr{E}}} \pi^{*}(x) \xi\right) .
\end{aligned}
$$

Hence, $P_{\overline{\mathscr{C}}} \xi \in \mathscr{D}\left(\pi_{\mathscr{E}}\right)^{*}$ and $\left(\pi_{\mathscr{E}}\right)^{*}(x) P_{\overline{\mathscr{C}}} \xi=P_{\overline{\mathscr{C}}} \pi^{*}(x) \xi$. The rest is clear.

Theorem 3.3. Let $\pi$ be a closed *-representation of a *algebra $\mathscr{A}$ on a Hilbert space $\mathscr{H}, \mathscr{E}$ a $\pi$-invariant subspace of $\mathscr{D}(\pi)$, and $P_{\overline{\mathscr{E}}}$ the projection of $\mathscr{H}$ onto $\overline{\mathscr{E}}$. Then the following statements hold:

(i) $\pi_{\mathscr{E}}$ is selfadjoint iff $P_{\overline{\mathscr{C}}} \mathscr{D}\left(\pi^{*}\right)=\mathscr{D}\left(\pi_{\mathscr{C}}\right)$.

(ii) If $P_{\overline{\mathscr{E}}} \mathscr{D}(\pi) \subset \mathscr{D}(\pi)$ then $P_{\overline{\mathscr{C}}} \in \pi(\mathscr{A})_{\mathrm{w}}^{\prime}$.

In particular, if $\pi$ is selfadjoint then

(i') $\pi_{\mathscr{E}}$ is selfadjoint iff $\pi_{P_{\overline{\mathscr{G}}}}=\pi_{\mathscr{E}}$;

(ii') $P_{\overline{\mathscr{E}}} \mathscr{D}(\pi) \subset \mathscr{D}(\pi)$ iff $P_{\overline{\mathscr{E}}} \in \pi(\mathscr{A})_{\mathrm{w}}^{\prime}$.

Proof. (i) Suppose $P_{\overline{\mathscr{C}}} \mathscr{D}\left(\pi^{*}\right)=\mathscr{D}\left(\pi_{\mathscr{C}}\right)$, and take an arbitrary $\xi$ in $\mathscr{D}\left(\pi_{\mathscr{E}}\right)^{*}$. Then it follows from Lemma 3.2(ii) that

$$
(\pi(x) \eta \mid \xi)=\left(P_{\overline{\mathscr{C}}} \pi(x) \eta \mid \xi\right)=\left(\pi_{\mathscr{E}}(x) P_{\overline{\mathscr{C}}} \eta \mid \xi\right)=\left(\eta \mid P_{\overline{\mathscr{E}}}\left(\pi_{\mathscr{E}}\right)^{*}\left(x^{*}\right) \xi\right)
$$


for each $x \in \mathscr{A}$ and $\eta \in \mathscr{D}(\pi)$. Hence $\xi=P_{\overline{\mathscr{E}}} \xi \in P_{\overline{\mathscr{C}}} \mathscr{D}\left(\pi^{*}\right)=\mathscr{D}\left(\pi_{\mathscr{E}}\right)$. Therefore, $\pi_{\mathscr{E}}$ is selfadjoint. The converse is clear from Lemma 3.2.

(ii) If $P_{\overline{\mathscr{C}}} \mathscr{D}(\pi) \subset \mathscr{D}(\pi)$, then for each $x \in \mathscr{A}$ and $\xi, \eta \in \mathscr{D}(\pi)$, using Lemma 3.2(ii) several times, we have

$$
\begin{aligned}
\left(P_{\overline{\mathscr{C}}} \pi(x) \xi \mid \eta\right) & =\left(P_{\overline{\mathscr{C}}} \pi(x) P_{\overline{\mathscr{C}}} \xi \mid \eta\right)+\left(P_{\overline{\mathscr{C}}} \pi(x)\left(I-P_{\overline{\mathscr{C}}}\right) \xi \mid \eta\right) \\
& \left.=\left(P_{\overline{\mathscr{C}}} \pi(x) P_{\overline{\mathscr{C}}} \xi \mid \eta\right)+\left(\pi_{\mathscr{C}}\right)^{*}(x) P_{\overline{\mathscr{C}}}\left(I-P_{\overline{\mathscr{C}}}\right) \xi \mid \eta\right) \\
& =\left(\xi \mid P_{\overline{\mathscr{C}}} \pi\left(x^{*}\right) P_{\overline{\mathscr{C}}} \eta\right)=\left(\xi \mid\left(\pi_{\mathscr{C}}\right)^{*}\left(x^{*}\right) P_{\overline{\mathscr{C}}} \eta\right) \\
& =\left(\xi \mid P_{\overline{\mathscr{C}}} \pi\left(x^{*}\right) \eta\right)=\left(P_{\overline{\mathscr{C}}} \xi \mid \pi\left(x^{*}\right) \eta\right) .
\end{aligned}
$$

(i'), (ii') These follow from the above statements (i), (ii).

Corollary 3.4. Let $\mathscr{E}$ be a $\pi$-invariant subspace of $\mathscr{D}(\pi)$ such that $P_{\overline{\mathscr{E}}}$ is contained in $\pi(\mathscr{A})_{\mathrm{w}}^{\prime}$. Then the following statements hold:

(i) $\pi_{\mathscr{E}} \subset \pi_{P_{\overline{\mathscr{G}}}} \subset \pi_{P_{\overline{\mathscr{g}}}}^{*} \subset\left(\pi_{\mathscr{E}}\right)^{*}, \pi \subset \pi_{P_{\overline{\mathscr{E}}}} \oplus \pi_{I-P_{\overline{\mathscr{G}}}} \subset \pi^{*}$.

(ii) $P_{\overline{\mathscr{E}}} \mathscr{D}(\pi) \subset \mathscr{D}(\pi)$ iff $\pi=\pi_{P_{\overline{\mathscr{E}}}} \oplus \pi_{I-P_{\overline{\mathscr{E}}}}$.

(iii) If $\pi$ is selfadjoint, then $\pi_{P_{\bar{E}}}$ and $\pi_{I-P_{\bar{E}}}$ are both selfadjoint and $\pi=$ $\pi_{P_{\overline{\mathscr{g}}}} \oplus \pi_{I-P_{\overline{\mathrm{g}}}}$.

Proof. (i) By Lemma 3.2 we have

$$
\mathscr{D}\left(\pi_{\mathscr{E}}\right) \subset P_{\overline{\mathscr{E}}} \mathscr{D}(\pi) \subset \mathscr{D}(\pi) \text { and } \pi_{\mathscr{E}}(x) \xi=P_{\overline{\mathscr{E}}} \pi(x) \xi=\pi(x) P_{\overline{\mathscr{C}}} \xi
$$

for each $x \in \mathscr{A}$ and $\xi \in \mathscr{D}(\pi)$. Hence, $\pi_{\mathscr{E}} \subset \pi_{P_{\overline{\mathscr{E}}}}$, and the rest is clear.

(ii) This follows from [7, Lemma 8.3.3].

(iii) Selfadjointness of $\pi$ and (3.1) imply that $\pi_{P_{\bar{\varepsilon}}}$ and $\pi_{I-P_{\bar{\varepsilon}}}$ are selfadjoint and $P_{\overline{\mathscr{G}}} \mathscr{D}(\pi) \subset \mathscr{D}(\pi)$. Therefore $\pi=\pi_{P_{\overline{\mathscr{g}}}} \oplus \pi_{I-P_{\overline{\bar{g}}}}$.

Remark 3.5. (1) The converse of Theorem 3.3(ii) and the equations in Corollary 3.4(i) do not necessarily hold (see Example 3.6).

(2) If $\pi$ is selfadjoint, then $\pi_{P}$ is always selfadjoint; but it is not true in case of $\pi_{\mathscr{C}}$. For instance, we have the result that there exists a weakly cyclic but not cyclic vector $\xi_{0}$ for a selfadjoint representation $\pi$ of $*$-algebra $\mathscr{A}[7$, Example 8.3.18]. Then $\mathscr{E}:=\pi(\mathscr{A}) \xi_{0}$ is a $\pi$-invariant subspace of $\mathscr{D}(\pi)$ and $\pi_{\mathscr{E}}$ is not selfadjoint.

(3) Schmüdgen has called a subspace $\mathscr{E}$ (resp. closed subspace $\overline{\mathscr{E}}$ ) of $\mathscr{D}(\pi)$ (resp. $\mathscr{H}(\pi)$ ) reducing for $\pi$ if the equation in Corollary 3.4(ii) holds [7, Definition 8.3.2]. And he has obtained the same results as Corollary 3.4(2) [7, Lemma 8.3.3].

Example 3.6. For a $*$-algebra $\mathscr{A}$ generated by a hermitian element $x_{0}$ and unit, we define a closed $*$-representation of $\mathscr{A}$ on Hilbert space $\mathscr{H}:=L^{2}([0,2])$,

$$
\begin{aligned}
\mathscr{D}(\pi) & :=\left\{f \in C^{\infty}([0,2]) ; f^{(n)}(0)=f^{(n)}(1)=f^{(n)}(2), n=0,1, \ldots\right\}, \\
\pi\left(x_{0}\right) f & :=-i(d / d t) f \text { for } f \in \mathscr{D}(\pi) .
\end{aligned}
$$

We put

$$
\mathscr{E}:=\left\{f \in C^{\infty}([0,2]) ; f^{(n)}(0)=f^{(n)}(t)=0, \forall t \in[1,2], n=0,1, \ldots\right\} .
$$

Then $\mathscr{E}$ is a $\pi$-invariant subspace of $\mathscr{D}(\pi)$ and the projection $P_{\overline{\mathscr{C}}}$ of $\mathscr{H}$ onto $\overline{\mathscr{E}}$ is contained in $\pi(\mathscr{A})_{\mathrm{w}}^{\prime}$, but $P_{\overline{\mathscr{C}} \mathscr{D}}(\pi) \not \subset \mathscr{D}(\pi)$. This is clear because

$$
\left(P_{\overline{\mathscr{E}}} f\right)(t)=\left\{\begin{array}{ll}
f(t) & (0 \leq t \leq 1), \\
0 & (1<t \leq 2)
\end{array} \text { for } f \in L^{2}([0,2]) .\right.
$$


Furthermore, $\pi_{\mathscr{E}}$ (resp. $\pi_{P_{\overline{\mathscr{E}}}}, \pi_{I-P_{\overline{\mathscr{E}}}}$ ) is unitarily equivalent to a nonselfadjoint *-representation $\pi_{0}$ (resp. selfadjoint representations $\pi_{1}, \pi_{2}$ ) of $\mathscr{A}[3,4]$, defined by

$$
\begin{aligned}
\mathscr{D}\left(\pi_{0}\right) & :=\left\{f \in C^{\infty}([0,1]) ; f^{(n)}(0)=f^{(n)}(1)=0, n=0,1, \ldots\right\}, \\
\mathscr{D}\left(\pi_{1}\right) & :=\left\{f \in C^{\infty}([0,1]) ; f^{(n)}(0)=f^{(n)}(1), n=0,1, \ldots\right\}, \\
\mathscr{D}\left(\pi_{2}\right) & :=\left\{f \in C^{\infty}([1,2]) ; f^{(n)}(1)=f^{(n)}(2), n=0,1, \ldots\right\}, \\
\pi_{j}\left(x_{0}\right) f & :=-i(d / d t) f \text { for } f \in \mathscr{D}\left(\pi_{j}\right) \text { and } j=0,1,2 .
\end{aligned}
$$

Hence we have

$$
\pi_{\mathscr{E}} \varsubsetneqq \pi_{P_{\overline{\mathscr{E}}}} \text { and } \pi \varsubsetneqq \pi_{P_{\overline{\mathscr{E}}}} \oplus \pi_{I-P_{\overline{\mathscr{E}}}}
$$

\section{A DECOMPOSITION OF CLOSED *-REPRESENTATIONS $\pi$}

Using the results of $\S 3$, we decompose a closed $*$-representation $\pi$. First we define some notions for vectors in $\mathscr{D}(\pi)$.

Definition 4.1. A vector $\varphi \in \mathscr{D}(\pi)$ is said to be a selfadjoint (resp. integrable, bounded) vector for $\pi$, if $\pi_{\mathscr{E}_{\varphi}}$ is a selfadjoint (resp. integrable, bounded) representation of $\mathscr{A}$, where $\mathscr{E}_{\varphi}:=\pi(\mathscr{A}) \varphi$.

Theorem 4.2. For any closed $*$-representation $\pi$ of $\mathscr{A}$, we have the decompositions

$$
\pi=\pi_{1}^{s} \oplus \pi_{2}^{s}=\pi_{1}^{i} \oplus \pi_{2}^{i}=\pi_{1}^{b} \oplus \pi_{2}^{b},
$$

where $\pi_{1}^{s}$ (resp. $\pi_{1}^{i}, \pi_{1}^{b}$ ) is a direct sum of selfadjoint (resp. integrable, bounded) representations of $\mathscr{A}$ with a cyclic vector and $\pi_{2}^{s}$ (resp. $\pi_{2}^{i}, \pi_{2}^{b}$ ) is a closed *representation of $\mathscr{A}$ that does not have any nonzero selfadjoint (resp. integrable, bounded) vector in the domain. Then $\pi_{1}^{b}$ is a *-subrepresentation of $\pi_{1}^{i}$ and $\pi_{1}^{i}$ is a *-subrepresentation of $\pi_{1}^{s}$.

Proof. Let $\left\{\varphi_{\lambda}\right\}_{\lambda \in \Lambda}$ be a maximal family of selfadjoint vectors for $\pi$ such that $\overline{\mathscr{E}_{\varphi_{\lambda}}} \perp \overline{\mathscr{E}_{\varphi_{u}}}$ for each $\lambda(\neq) \mu \in \Lambda$. Then $\left\{P_{\lambda}:=\operatorname{proj} \overline{\mathscr{E}}_{\varphi_{\lambda}}\right\}$ is a family of mutually orthogonal projections in $\pi(\mathscr{A})_{\mathrm{w}}^{\prime}$. We put $P_{1}:=\sum_{\lambda \in \Lambda} P_{\lambda}, P_{2}:=I-P_{1}$. Since $\pi(\mathscr{A})_{\mathrm{w}}^{\prime}$ is weakly closed, we have

$$
P_{1}, P_{2} \in \pi(\mathscr{A})_{\mathrm{w}}^{\prime}, \quad P_{1} \mathscr{D}(\pi) \subset \mathscr{D}(\pi), \quad P_{2} \mathscr{D}(\pi) \subset \mathscr{D}(\pi),
$$

and so, $\pi=\pi_{P_{1}} \oplus \pi_{P_{2}}$. Then $\pi_{P_{1}}$ is a direct sum of a family of the selfadjoint representations $\pi_{P_{\lambda}}$ of $\mathscr{A}$ with cyclic vector $\varphi_{\lambda}$. We show that $\pi_{P_{2}}$ does not have any nonzero selfadjoint vector. Suppose that some nonzero vector $\varphi$ in $\mathscr{D}\left(\pi_{P_{2}}\right)$ is selfadjoint for $\pi_{P_{2}}$. Then, since $P_{2} \in \pi(\mathscr{A})_{\mathrm{w}}^{\prime}$ and $\varphi=P_{2} \varphi \in$ $\mathscr{D}\left(\pi_{P_{2}}\right)$, it follows that

$$
\pi_{P_{2}}(\mathscr{A}) \varphi=\pi(\mathscr{A}) P_{2} \varphi=\pi(\mathscr{A}) \varphi,
$$

which implies that $\varphi$ is a selfadjoint vector for $\pi$. This contradicts the maximality of $\left\{\varphi_{\lambda}\right\}_{\lambda \in \Lambda}$. Thus, we have the decomposition of $\pi$ in theorem, as $\pi_{2}^{s}:=\pi_{P_{2}}$ and $\pi_{1}^{s}:=\pi_{P_{1}}$. Similarly we can show that $\pi=\pi_{1}^{i} \oplus \pi_{2}^{i}$ and $\pi=\pi_{1}^{b} \oplus \pi_{2}^{b}$.

Example 4.3. We denote by $C^{\infty}(\mathbf{R})$ the set of all infinitely differentiable functions on $\mathbf{R}$ and

$$
C_{0}^{\infty}(\mathbf{R}):=\left\{f \in C^{\infty}(\mathbf{R}) ; \operatorname{supp} f \text { is compact in } \mathbf{R}\right\} .
$$


Let $\mathscr{A}$ be a $*$-algebra generated by two hermitian generators $p$ and $q$ satisfying the commutation relation $p q-q p=-i I,{ }^{1}$ and let $\pi_{0}$ be a $*$-representation of $\mathscr{A}$ on the Hilbert space $L^{2}(\mathbf{R})$ with the domain $C_{0}^{\infty}(\mathbf{R})$,

$$
\begin{aligned}
& \left(\pi_{0}(p) f\right)(t)=-i d f / d t, \\
& \left(\pi_{0}(q) f\right)(t)=t f(t), \quad f \in C_{0}^{\infty}(\mathbf{R}), t \in \mathbf{R} .
\end{aligned}
$$

Then the closure $\pi$ of $\pi_{0}$ is the Schrödinger representation of $\mathscr{A}$, and it is well known that $\pi$ is a selfadjoint representation with a cyclic vector $\varphi_{0}(t)=$ $\pi^{-1 / 4} \exp \left(-t^{2} / 2\right)$ and $\pi(\mathscr{A})_{\mathrm{w}}^{\prime}=\mathrm{CI}[3,6]$. Hence $\pi$ has a selfadjoint vector $\varphi_{0}$, but $\pi_{0}$ does not have any nonzero selfadjoint vector.

Example 4.4. We next consider an $\mathscr{O}^{*}$-algebra $\mathscr{M}_{1}$ on $C_{0}^{\infty}(\mathbf{R})$ that contains the preceding $\pi_{0}(\mathscr{A})$. We put

$$
\mathscr{M}_{1}=\left\{\left.\sum_{k=1}^{n} f_{k}\left(\frac{d}{d t}\right)^{k}\right|_{C_{0}^{\infty}(\mathbf{R})} ; f_{k} \in C^{\infty}(\mathbf{R}), 0 \leq k \leq n, n \in \mathbf{N}\right\},
$$

and by the identity mapping on $\mathscr{M}_{1}$ we define a *-representation $\pi_{1}$ of $\mathscr{M}_{1}$ on $L^{2}(\mathbf{R})$ with the domain $C_{0}^{\infty}(\mathbf{R})$. Then $\pi_{1}$ is selfadjoint and any nonzero vector in $C_{0}^{\infty}(\mathbf{R})$ is not a selfadjoint vector for $\pi_{1}$. In fact, by the preceding discussion, we have

$$
\begin{gathered}
\mathscr{D}\left(\pi_{1}^{*}\right)=\mathscr{D}^{*}\left(\mathscr{M}_{1}\right) \subset \mathscr{D}^{*}\left(\pi_{0}(\mathscr{A})\right)=\mathscr{S}(\mathbf{R}), \\
\left(\mathscr{M}_{1}\right)_{\mathrm{w}}^{\prime} \subset\left(\pi_{0}(\mathscr{A})\right)_{\mathrm{w}}^{\prime}=\pi(\mathscr{A})_{\mathrm{w}}^{\prime}=\mathbf{C I} .
\end{gathered}
$$

For an arbitrary $\varphi \in \mathscr{D}\left(\pi_{1}^{*}\right)$, it is in $\mathscr{S}(\mathbf{R})$ and $\bar{f} \varphi \in L^{2}(\mathbf{R})$ for $f \in C^{\infty}$, hence $\varphi \in C_{0}^{\infty}(\mathbf{R})=\mathscr{D}\left(\pi_{1}\right)$. Therefore $\pi_{1}$ is selfadjoint. Suppose there is a nonzero selfadjoint vector $\varphi_{0}$ in $C_{0}^{\infty}(\mathbf{R})$, then we have $P_{\varphi_{0}} \in \pi(\mathscr{A})_{\mathrm{w}}^{\prime}, P_{\varphi_{0}} \neq 0$, and $P_{\varphi_{0}} \neq I$. This contradicts (4.2).

Example 4.5. The following simple example shows that there is a representation whose domain consists of (non)selfadjoint vectors. Let $\mathscr{D}$ be a dense subspace of a Hilbert space $\mathscr{H}$ such that $\mathscr{L}^{\dagger}(\mathscr{D})$ is closed. Hence, each $\varphi \in \mathscr{D}$ is ultracyclic for $\mathscr{L}^{\dagger}(\mathscr{D})$ (i.e., $\left.\mathscr{L}^{\dagger}(\mathscr{D}) \varphi=\mathscr{D}\right)$. Therefore, if $\mathscr{L}^{\dagger}(\mathscr{D})$ is selfadjoint (resp. nonselfadjoint) then each $\varphi$ in $\mathscr{D}$ is a selfadjoint (resp. nonselfadjoint) vector for $\mathscr{L}^{\dagger}(\mathscr{D})$.

Example 4.6. We call a $*$-representation $\pi$ of $\mathscr{A}$ weakly unbounded, if $\pi$ is decomposed into a direct sum of bounded representations of $\mathscr{A}$. Then we have the following statements.

Statement 1. If $\pi$ is weakly unbounded, then $\pi$ is integrable and each vector in $\mathscr{D}(\pi)$ is integrable for $\pi$.

Statement 2 . Let $\mathscr{A}$ be a $*$-algebra generated by finite commutative hermitian generators $\left\{a_{1}, a_{2}, \ldots, a_{n}\right\}$ and $\pi$ a closed *-representation of $\mathscr{A}$. Then $\pi$ is integrable iff $\pi$ is weakly unbounded.

Proof. Suppose $\pi=\bigoplus_{\lambda \in \Lambda} \pi_{\lambda}$ is weakly unbounded, then it is clear that $\pi$ is integrable. For an arbitrary $\varphi=\left(\varphi_{\lambda}\right) \in \mathscr{D}(\pi)$, we have $\pi_{\mathscr{\varepsilon}_{\varphi}}=\bigoplus_{\lambda \in \Lambda}\left(\pi_{\lambda}\right)_{\mathscr{\varepsilon}_{\varphi_{\lambda}}}$, and

\footnotetext{
${ }^{1}$ The referee pointed out that this decomposition is also obtained by [7, Corollary 8.3.13].
} 
so $\pi_{\mathscr{E}_{\varphi}}$ is weakly unbounded. Therefore, $\varphi$ is integrable for $\pi$. This completes the proof of Statement 1. To show Statement 2, suppose $\pi$ is integrable. Then, for each $k=1,2, \ldots, n, \overline{\pi\left(a_{k}\right)}$ is selfadjoint, and so we have the spectral resolutions $\overline{\pi\left(a_{k}\right)}=\int \lambda d P_{k}(\lambda) .\left\{P_{k}(\lambda) ; \lambda \in \mathbf{R}, k=1,2, \ldots, n\right\}$ are mutually commuting [6], and we have the following mutually orthogonal family,

$$
\left\{P_{k}^{m}:=P_{k}\left(\lambda_{m-1}, \lambda_{m}\right]: m \in \mathbf{N}\right\} ; \quad \sum_{m \in \mathbf{N}} P_{k}^{m}=I
$$

for $k=1,2, \ldots, n$. Furthermore we can take $\left\{P_{k}^{m_{k}} \in\left\{P_{k}^{m}\right\}: k=1,2, \ldots\right.$, $n\}$, satisfying

$$
P_{1}^{m_{1}} P_{2}^{m_{2}} \cdots P_{n}^{m_{n}} \neq 0
$$

Since $\left\{P_{k}^{m_{k}}\right\} \subset \pi(\mathscr{A})_{\mathrm{w}}^{\prime}$ and $\pi(\mathscr{A})_{\mathrm{w}}^{\prime} \mathscr{D}(\pi) \subset \mathscr{D}(\pi)$, there is a vector $\varphi$ in $\mathscr{D}(\pi)$ such that $P_{1}^{m_{1}} P_{2}^{m_{2}} \cdots P_{n}^{m_{n}} \varphi=\varphi$. From this, $\varphi$ is a bounded vector for $\pi$, and so $\pi_{2}^{b}=0$ in Theorem 4.2. Therefore, $\pi=\pi_{1}^{b}$, that is, $\pi$ is weakly unbounded.

Example 4.7. In this example we deal with the Hilbert space of all HilbertSchmidt operators on a separable Hilbert space. Let $\mathscr{H}$ be a separable Hilbert space and $\mathscr{D}$ a dense subspace of $\mathscr{H}$ such that $\mathscr{L}^{\dagger}(\mathscr{D})$ is closed. We denote by $\mathscr{H} \otimes \overline{\mathscr{H}}$ the Hilbert space of all Hilbert-Schmidt operators on $\mathscr{H}$, with inner product $\langle\cdot \mid \cdot\rangle$ and we put,

$$
\begin{aligned}
\mathscr{D} \otimes \overline{\mathscr{H}} & :=\{T \in \mathscr{H} \otimes \overline{\mathscr{H}} ; T \mathscr{H} \subset D\}, \\
\sigma_{2}(\mathscr{D}) & :=\left\{T \in \mathscr{D} \otimes \overline{\mathscr{H}} ; X T \in \mathscr{H} \otimes \overline{\mathscr{H}} \text { for each } X \in \mathscr{L}^{\dagger}(\mathscr{D})\right\},
\end{aligned}
$$

then $\sigma_{2}(\mathscr{D})$ is a dense subspace of $\mathscr{H} \otimes \overline{\mathscr{H}}$. Thus we have a closed $*$-representation $\pi$ of $\mathscr{L}^{\dagger}(\mathscr{D})$ on $\mathscr{H} \otimes \overline{\mathscr{H}}$,

$$
\begin{aligned}
\mathscr{D}(\pi) & :=\sigma_{2}(\mathscr{D}), \\
\pi(X) T & :=X T, \quad X \in \mathscr{L}^{\dagger}(\mathscr{D}), \quad T \in \mathscr{D}(\pi) .
\end{aligned}
$$

We now consider the selfadjointness of elements in $\mathscr{D}(\pi)$. For this, we remark that a nonsingular element $\Omega$ in $\sigma_{2}(\mathscr{D})$ is represented as $\Omega=\sum_{n=1}^{\infty} \lambda_{n} e_{n} \otimes \overline{e_{n}}$, for some orthonormal system $\left\{e_{n}\right\}$ in $\mathscr{H}$ and $\left\{\lambda_{n} \neq 0\right\} \subset \mathbf{C}$, where $(\varphi \otimes \bar{\psi}) \phi=$ $(\phi \mid \psi) \varphi$ for $\varphi, \psi, \phi, \in \mathscr{H}$. Then we have the following statements.

Statement 1. Let

$$
P_{\Omega}:=\operatorname{proj} \overline{\pi\left(\mathscr{L}^{\dagger}(\mathscr{D})\right) \Omega}, \quad \mathscr{E}_{\Omega}:=\pi\left(\mathscr{L}^{\dagger}(\mathscr{D})\right) \Omega,
$$

where $\Omega=\sum_{n=1}^{\infty} \lambda_{n} e_{n} \otimes \overline{e_{n}} \in \mathscr{D}(\pi)=\sigma_{2}(\mathscr{D}) \quad\left(\left\{\lambda_{n} \neq 0\right\}\right)$. Then, $P_{\Omega} \in \pi\left(\mathscr{L}^{\dagger}(\mathscr{D})\right)_{\mathrm{w}}^{\prime}$ and $\pi_{\mathscr{E}_{\Omega}}=\pi_{P_{\Omega}}$.

Statement 2. A nonsingular element $\Omega=\sum \lambda_{n} e_{n} \otimes \overline{e_{n}}$ is selfadjoint for $\pi$ iff

$$
\left\{T_{e_{k}} ; k \in \mathbf{N}, T \in \mathscr{D}\left(\pi^{*}\right)\right\} \subset \mathscr{D} .
$$

Statement 3. If $\mathscr{L}^{\dagger}(\mathscr{D})$ is selfadjoint, then an arbitrary nonsingular $\Omega \in \mathscr{D}(\pi)$ is selfadjoint for $\pi$. If $\mathscr{L}^{\dagger}(\mathscr{D})$ is not selfadjoint, then no nonsingular element of $\sigma_{2}(\mathscr{D})$ is selfadjoint for $\pi$. 
Proof. Let $\pi^{\prime}(A) T:=T A$ for $A \in \mathscr{B}(\mathscr{H}), T \in \mathscr{H} \otimes \overline{\mathscr{H}}$. Then we have

$$
P_{\Omega}=\pi^{\prime}\left(\sum e_{n} \otimes \overline{e_{n}}\right) \text {. }
$$

In fact, for each $T \in\left(I-P_{\Omega}\right) \mathscr{H} \otimes \overline{\mathscr{H}}, k \in \mathbf{N}$, and $\varphi \in \mathscr{D}$, we have

$$
\begin{aligned}
0 & =\left\langle T \mid \pi\left(\varphi \otimes \overline{e_{k}}\right) \Omega\right\rangle=\left\langle T \mid \lambda_{k}\left(\varphi \otimes \overline{e_{k}}\right)\right\rangle \\
& =\sum_{j \in \mathbf{N}}\left(T e_{j} \mid \lambda_{k}\left(\varphi \otimes \overline{e_{k}}\right) e_{j}\right) \\
& =\left(T e_{k} \mid \lambda_{k}\left(\varphi \otimes \overline{e_{k}}\right) e_{k}\right)=\left(T_{e_{k}} \mid \lambda_{k} \varphi\right),
\end{aligned}
$$

since $\lambda_{k} \neq 0$, hence $T e_{k}=0$ and

$$
\pi^{\prime}\left(\sum e_{k} \otimes \overline{e_{k}}\right) T=T \sum e_{k} \otimes \overline{e_{k}}=\sum T e_{k} \otimes \overline{e_{k}}=0 .
$$

Thus $T \in\left(I-\pi^{\prime}\left(\sum e_{k} \otimes \overline{e_{k}}\right)\right) \mathscr{H} \otimes \overline{\mathscr{H}}$. Therefore, $P_{\Omega} \geq \pi^{\prime}\left(\sum e_{k} \otimes \overline{e_{k}}\right)$. Since $\Omega\left(\sum e_{k} \otimes \overline{e_{k}}\right)=\Omega$, the converse inequality holds. Furthermore, we have

$$
\mathscr{D}\left(\pi_{\mathscr{C}_{\Omega}}\right)=P_{\Omega} \mathscr{D}(\pi) \text {. }
$$

To prove this, for each $S \in \mathscr{D}(\pi)$ we put

$$
A_{n}:=\sum_{k=1}^{n} \lambda_{k}^{-1} e_{k} \otimes \overline{e_{k}}, \quad S_{n}:=\left.S A_{n}\right|_{\mathscr{D}}\left(\in \mathscr{L}^{\dagger}(\mathscr{D})\right), \quad n \in \mathbf{N},
$$

then from (4.3),

$$
\begin{aligned}
& \left(\left\|\pi(X) \pi\left(S_{n}\right) \Omega-\pi(X) P_{\Omega} S\right\|_{2}\right)^{2} \\
& =\left(\left\|X S_{n} \Omega-X P_{\Omega} S\right\|_{2}\right)^{2}=\left(\left\|X S \sum_{k=1}^{n} e_{k} \otimes \overline{e_{k}}-X S \sum_{k=1}^{\infty} e_{k} \otimes \overline{e_{k}}\right\|_{2}\right)^{2} \\
& =\left(\left\|X S \sum_{k=n+1}^{\infty} e_{k} \otimes \overline{e_{k}}\right\|_{2}\right)^{2}=\sum_{k=n+1}^{\infty}\left(\left\|X S e_{k}\right\|\right)^{2} \rightarrow 0 \quad(n \rightarrow \infty),
\end{aligned}
$$

for each $X \in \mathscr{L}^{\dagger}(\mathscr{D})$, where $\|\cdot\|_{2}$ is a norm defined by $\langle\cdot \mid \cdot\rangle$. Hence, $P_{\Omega} S \in$ $\overline{\pi\left(\mathscr{L}^{\dagger}(\mathscr{D})\right) \Omega^{t_{\pi}}}$. Therefore, $P_{\Omega} \mathscr{D}(\pi) \subset \mathscr{D}\left(\pi_{\mathscr{C}_{\Omega}}\right)$. Lemma 3.2(i) implies the converse. From (4.4), Statement 1 is clear.

It is sufficient to prove for Statement 2 that $P_{\Omega} \mathscr{D}\left(\pi^{*}\right)=P_{\Omega} \mathscr{D}(\pi)$ if $\left\{T e_{k}\right\} \subset$ $\mathscr{D}$. Suppose $\left\{T e_{k} ; k \in \mathbf{N}, T \in \mathscr{D}\left(\pi^{*}\right)\right\} \subset \mathscr{D}$. For each $T \in \mathscr{D}\left(\pi^{*}\right)$ we put $S:=T\left(\sum e_{j} \otimes \overline{e_{j}}\right)$. Then we have $S \mathscr{H} \subset \mathscr{D}$ and $X S \in \mathscr{H} \otimes \overline{\mathscr{H}}$ for each $X \in \mathscr{L}^{\dagger}(\mathscr{D})$. Hence, $P_{\Omega} T=S \in \mathscr{D}(\pi)$ and $P_{\Omega} \mathscr{D}\left(\pi^{*}\right)=P_{\Omega} \mathscr{D}(\pi)$.

We show Statement 3 . Let $\Omega=\sum \lambda_{n} e_{n} \otimes \bar{e}_{n}$ be an arbitrarily nonsingular element of $\mathscr{L}^{\dagger}(\mathscr{D})$. Suppose $\mathscr{L}^{\dagger}(\mathscr{D})$ is selfadjoint. Then it is easily shown that $\pi$ is selfadjoint, which implies $\left\{T e_{k} ; k \in \mathbf{N}, T \in \mathscr{D}\left(\pi^{*}\right)\right\} \subset \mathscr{D}$. Hence it follows from Statement 2 that $\Omega$ is a selfadjoint vector for $\pi$.

Suppose $\mathscr{L}^{\dagger}(\mathscr{D})$ is not selfadjoint. Then there is a nonzero element $\varphi$ of $\mathscr{D}^{*}\left(\mathscr{L}^{\dagger}(\mathscr{D})\right):=\bigcap_{X \in \mathscr{L}^{\dagger}(\mathscr{D})} \mathscr{D}\left(X^{*}\right)$ such that $\varphi \notin \mathscr{D}$. And putting $T:=\varphi \otimes \bar{\psi}$ for nonzero $\psi \in \mathscr{H}$, we have $T \in \mathscr{D}\left(\pi^{*}\right)$ but $T e_{k} \notin \mathscr{D}$ for some $k \in \mathbf{N}$. Hence $\Omega$ is not selfadjoint for $\pi$.

\section{ACKNOWLEDGMENT}

We thank Dr. H. Kurose for many helpful discussions concerning this work. 


\section{REFERENCES}

1. I. M. Gelfand and N. Ya. Vilenkin, Generalized functions, Vol. 4, Academic Press, New York, 1964.

2. S. P. Gudder and W. Scruggs, Bounded representations of *-algebras, Pacific J. Math. 70 (1977), 369-382.

3. S. P. Gudder and R. L. Hudson, A non commutative probability theory, Trans. Amer. Math. Soc. 245 (1978), 1-41.

4. A. Inoue and K. Takesue, Self-adjoint representations of polynomials algebras, Trans. Amer. Math. Soc. 280 (1983), 393-400.

5. P. E. T. Jorgensen and R. T. Moore, Operator commutation relations, Reidel, Dordrecht and Boston, MA, 1984.

6. R. T. Powers, Self-adjoint algebras of unbounded operators. I, Comm. Math. Phys. 21 (1971), 85-124.

7. K. Schmüdgen, Unbounded operator algebras and representation theory, Birkhäuser Verlag, Basel, Boston, and Berlin, 1990.

Department of Applied Mathematics, Fukuoka University, Fukuoka, 814-01, Japan 